\title{
Association of Hemoglobin and Blood Pressure in a Chinese Community-Dwelling Population
}

\author{
Yan Xuan ${ }^{a}$ Junli Zuo ${ }^{a}$ Shuping Zheng ${ }^{c}$ Jinbao $J^{c} \quad Y^{c}$ Qian $^{b}$ \\ a Department of Geriatrics, Ruijin Hospital North, Shanghai Jiaotong University Medicine \\ School, Shanghai, China; 'bepartment of Respiratory Medicine, Ruijin Hospital North, \\ Shanghai Jiaotong University School of Medicine, Shanghai, China; ' Jiading District \\ Jiangqiao Community Health Service Center, Shanghai, China
}

\section{Keywords}

Hemoglobin · Systolic blood pressure · Normal glucose metabolism · Chinese population

\begin{abstract}
This study investigated the relationship between hemoglobin $(\mathrm{Hb})$ level and blood pressure in a Chinese community-dwelling population with normal glucose metabolism. $\mathrm{Hb}$, fasting plasma glucose, glycated $\mathrm{Hb} \mathrm{A1c}$, hepatic and renal function, lipid, electrolytes, and anthropometric parameters were measured. $\mathrm{Hb}$ level was found to be positively correlated with systolic (SBP; $r=0.075, p<0.001$ ) and diastolic (DBP; $r=0.272, p<0.001$ ) blood pressure in the cohort. The relationship was not affected by age, BMI, serum creatinine $(\mathrm{Cr})$, and low-density lipoprotein (LDL) in both males and females. Multivariate stepwise regression showed that age $(\beta=0.556, p<0.001), \mathrm{BMI}(\beta=1.107,<0.001), \mathrm{Hb}(\beta=0.082, p<0.001), \mathrm{Cr}(\beta=-0.032, p<$ $0.001)$, and $\operatorname{LDL}(\beta=1.023, p<0.001)$ were independent factors for SBP, and $\mathrm{Hb}(\beta=0.168$, $p<0.001)$, BMI $(\beta=0.519, p=0.001)$, and LDL $(\beta=0.331, p<0.001)$ for DBP. Hb level is positively associated with both SBP and DBP in a Chinese community-dwelling population with normal glucose metabolism.

(C) 2018 S. Karger AG, Basel
\end{abstract}

\section{Introduction}

Worldwide, high blood pressure is regarded as one of the most important underlying causes of cardiovascular disease $[1,2]$. It has been reported that $54 \%$ of the stroke cases and $47 \%$ of the ischemic heart disease cases could be attributed to a high blood pressure [2]. Mild 
Xuan et al.: Association of Hemoglobin and Blood Pressure in a Chinese

Community-Dwelling Population

changes in blood pressure of about 2-3 mm Hg can lead to fatal adverse events [3, 4]. Gaining knowledge about the cause of high blood pressure is important for getting it under control.

Some studies have shown that hemoglobin $(\mathrm{Hb})$ levels are related to blood pressure. Systolic blood pressure (SBP) and diastolic blood pressure (DBP) may increase with increasing $\mathrm{Hb}[5,6]$. Previous studies found that erythropoietin was related to an elevated blood pressure [7]. In previous studies, the relationship between $\mathrm{Hb}$ and blood pressure was found mainly in patient cohorts. There are few reports for the normal population. Atsma et al. [8] have shown that $\mathrm{Hb}$ level is positively associated with blood pressure in a large Dutch cohort of healthy individuals who were voluntary blood donors. The main objective of this study was to investigate the association between Hb levels and SBP and DBP in a Chinese communitydwelling cohort.

\section{Patients and Methods}

The study population was selected from the Xuhui District community of Shanghai, and subjects with normal glucose tolerance were studied. Normal glucose tolerance is defined as a plasma glucose level $\leq 126$ $\mathrm{mg} / \mathrm{dL}$ and glycated $\mathrm{Hb}$ A1c $(\mathrm{HbA1c}) \leq 6.5 \%$.

A physician administered a standardized questionnaire about each subject's medical history, intake of medications, and smoking and drinking habits. Nurses measured body height to the nearest $0.5 \mathrm{~cm}$. Participants wore light indoor clothing without shoes for body weight measurement. BMI was weight in kilograms divided by height in square meters. After the subjects had fasted overnight, venous blood samples were obtained and analyzed by automated enzymatic methods for serum total cholesterol and blood glucose. The presence of diabetes mellitus was assessed as a plasma glucose level $>126 \mathrm{mg} / \mathrm{dL}$ or HbA1c $\geq 6.5 \%$ [9] or the use of antidiabetic drugs.

Basic information included age, sex, diabetes mellitus, hypertension, smoking habits, and cardiovascular diseases. Venous blood and urine samples were obtained in a fasting state in the morning. Body weight, body height, waist circumference, and hip circumference were measured by the physician. Biological markers were assayed with standard laboratory techniques on colorimetric enzymatic assay systems.

Statistical Analysis

Spearman's bivariate correlation tests and partial correlation analyses were conducted to study the associations between blood pressure and anthropometric indices, FPG, HbA1c, and serum biochemical parameters with adjustment for confounders. Variables showing significant relationships with $\mathrm{Hb}$ and SBP/DBP in Spearman's correlations were used as independent variables in the multivariate stepwise linear regression models to test the combined effect of these independent factors on the dependent variables SBP or DBP. The Kolmogorov-Smirnoff test was employed to test the normality of the data. Group differences were compared with ANOVA tests for normally distributed variables, whereas the nonparametric Mann-Whitney U test was performed for skewed parameters. The results are presented as mean \pm SD. $p<0.05$ was considered significant. The statistical analysis was performed using SPSS 19.0 software (SPSS, Inc).

\section{Results}

Baseline Clinical Characteristics

The total cohort comprised 9,181 (3,888 males) subjects. The baseline anthropometric parameters and biochemical indices of the subjects are shown in Table 1.

\section{Association of Hb Level with SBP and DBP}

Spearman's correlation analysis revealed a significant positive correlation between the various clinical and biochemical parameters and SBP and DBP. SBP was also positively correlated with $\mathrm{Hb}(r=0.075, p<0.001)$ and DBP $(r=0.272, p<0.001)$ (Table 2$)$. 
Xuan et al.: Association of Hemoglobin and Blood Pressure in a Chinese

Community-Dwelling Population

Table 1. General characteristics of male and female subjects with normal glucose tolerance

\begin{tabular}{lccrr}
\hline & Total & Male & Female & $p$ value \\
\hline$N$ & 9,181 & 3,888 & 5,293 & $<0.001$ \\
Age, years & $61.45 \pm 12.35$ & $62.40 \pm 12.20$ & $60.76 \pm 12.41$ & $<0.001$ \\
BMI & $24.36 \pm 3.33$ & $24.63 \pm 3.27$ & $24.17 \pm 3.37$ & $<0.001$ \\
SBP, mm Hg & $134.99 \pm 19.33$ & $135.85 \pm 18.32$ & $134.35 \pm 20.02$ & $<0.001$ \\
DBP, mm Hg & $85.29 \pm 10.35$ & $87.35 \pm 10.51$ & $83.77 \pm 9.96$ & 0.007 \\
FDG, mm Hg & $4.85 \pm 0.58$ & $4.86 \pm 0.61$ & $4.85 \pm 0.56$ & 0.614 \\
HbA1c, \% & $5.52 \pm 0.41$ & $5.51 \pm 0.29$ & $5.53 \pm 0.42$ & 0.110 \\
Hb, g/L & $136.91 \pm 14.77$ & $147.47 \pm 12.17$ & $129.19 \pm 11.33$ & $<0.001$ \\
TC, mmol/L & $4.99 \pm 0.95$ & $4.78 \pm 0.92$ & $5.14 \pm 0.95$ & $<0.001$ \\
TG, mmol/L & $1.55 \pm 1.10$ & $1.59 \pm 1.19$ & $1.52 \pm 1.03$ & $<0.001$ \\
HDL, mmol/L & $1.41 \pm 0.36$ & $1.49 \pm 0.36$ & $1.29 \pm 0.33$ & $<0.001$ \\
LDL, mmol/L & $3.17 \pm 0.84$ & $3.05 \pm 0.81$ & $3.25 \pm 0.85$ & $<0.001$ \\
ALT, U/L & $18.91 \pm 14.49$ & $20.93 \pm 13.61$ & $17.43 \pm 14.93$ & $<0.001$ \\
AST, U/L & $21.12 \pm 9.74$ & $21.55 \pm 8.12$ & $20.81 \pm 10.77$ & $<0.001$ \\
AKP, U/L & $75.71 \pm 31.61$ & $75.45 \pm 33.01$ & $75.90 \pm 30.53$ & 0.508 \\
Cr, mmol/L & $71.77 \pm 20.94$ & $82.66 \pm 18.79$ & $63.77 \pm 18.71$ & $<0.001$ \\
UA, mmol/L & $316.85 \pm 83.35$ & $361.69 \pm 80.52$ & $283.91 \pm 68.75$ & $<0.001$ \\
\hline
\end{tabular}

The values are presented as mean $\pm \mathrm{SD}$, unless otherwise indicated. FPG, fasting plasma glucose; ALT, alanine transferase; AST, aspartate transferase; $\gamma$-GT, $\gamma$-glutamyltranspeptidase; AKP, alkaline phosphatase; UA, uric acid; TC, total cholesterol; TG, triglyceride.

Table 2. Correlations of SBP and DBP with various clinical and biochemical parameters

\begin{tabular}{lrrrrrr}
\hline & \multicolumn{2}{l}{ SBP } & & \multicolumn{2}{l}{ DBP } & \\
\cline { 2 - 3 } \cline { 6 - 7 } & $r$ & & $p$ value & & $r$ & $p$ value \\
\hline Age, years & 0.375 & $<0.001$ & & 0.003 & 0.763 \\
BMI & 0.256 & $<0.001$ & & 0.215 & $<0.001$ \\
Hb, g/L & 0.075 & $<0.001$ & & 0.272 & $<0.001$ \\
DBP, mm Hg & 0.587 & $<0.001$ & & 0.587 & $<0.001$ \\
FDG, mm Hg & 0.158 & $<0.001$ & & 0.080 & $<0.001$ \\
HbA1c, \% & 0.155 & $<0.001$ & & 0.023 & $<0.001$ \\
TC, mmol/L & 0.097 & $<0.001$ & & 0.137 & $<0.001$ \\
TG, mmol/L & 0.106 & $<0.001$ & & 0.052 & $<0.001$ \\
HDL, mmol/L & -0.067 & $<0.001$ & & -0.114 & $<0.001$ \\
LDL, mmol/L & 0.098 & $<0.001$ & & 0.056 & $<0.001$ \\
Cr, mmol/L & 0.069 & $<0.001$ & & 0.037 & 0.008 \\
\hline
\end{tabular}

FPG, fasting plasma glucose; TC, total cholesterol; TG, triglyceride.

$\mathrm{Hb}$ has a significant positive correlation with SBP and DBP, and the positive correlation persists after correcting for age, serum creatinine (Cr), BMI, and low-density lipoprotein (LDL) in males and females separately (Table 3, 4).

\section{Factors Determining Changes in SBP and DBP Levels in the Subjects}

To evaluate the independent relationship between $\mathrm{Hb}$ and blood pressure in males and females separately, we performed a stepwise multivariate regression analysis. Multivariate stepwise regression analysis revealed that among those factors that showed associations with $\mathrm{Hb}$, the major factors responsible for the changes in SBP were as follows: age ( $\beta=0.556$, $p<0.001$; BMI $(\beta=-1.107, p=0.000)$; $\mathrm{Hb}(\beta=0.082, p=0.013)$; $\mathrm{Cr}(\beta=-0.032, p<0.001)$; 
Table 3. Correlations of $\mathrm{Hb}$ with SBP and DBP after adjustment for confounding factors in the male population
Table 4. Correlations of $\mathrm{Hb}$ with SBP and DBP after adjustment for confounding factors in the female population
Xuan et al.: Association of Hemoglobin and Blood Pressure in a Chinese Community-Dwelling Population

\begin{tabular}{lll}
\hline & $r$ & $p$ value \\
\hline SBP & & \\
Model 1 & 0.110 & $<0.001$ \\
Model 2 & 0.062 & $<0.001$ \\
Model 3 & 0.057 & $<0.001$ \\
Model 4 & 0.052 & $<0.001$ \\
Model 5 & 0.058 & $<0.001$ \\
\hline DBP & & \\
Model 1 & 0.272 & $<0.001$ \\
Model 2 & 0.215 & $<0.001$ \\
Model 3 & 0.182 & $<0.001$ \\
Model 4 & 0.174 & $<0.001$ \\
Model 5 & 0.232 & $<0.001$ \\
\hline
\end{tabular}

Model 1: adjustment for age; Model 2: adjustment for age and $\mathrm{Cr}_{\text {; }}$ Model 3: adjustment for age, Cr, and BMI; Model 4: adjustment for age, CR, BMI, and LDL; Model 5: adjustment for age, Cr, BMI, LDL, and uric acid.

\begin{tabular}{lll}
\hline & $r$ & $p$ value \\
\hline SBP & & \\
Model 1 & 0.097 & $<0.001$ \\
Model 2 & 0.069 & $<0.001$ \\
Model 3 & 0.066 & $<0.001$ \\
Model 4 & 0.061 & $<0.001$ \\
Model 5 & 0.057 & $<0.001$ \\
\hline DBP & & \\
Model 1 & 0.265 & $<0.001$ \\
Model 2 & 0.197 & $<0.001$ \\
Model 3 & 0.170 & $<0.001$ \\
Model 4 & 0.165 & $<0.001$ \\
Model 5 & 0.221 & $<0.001$ \\
\hline
\end{tabular}

Model 1: adjustment for age; Model 2: adjustment for age and CR; Model 3: adjustment for age, $\mathrm{Cr}$, and BMI; Model 4: adjustment for age, CR, BMI, and LDL; Model 5: adjustment for age, Cr, BMI, LDL, and uric acid.

and LDL ( $\beta=1.032, p<0.001$ ) (adjusted $R^{2}$ for the model $=0.618, p=0.01$ ) (Table 3 ). When DBP was used as a dependent variable, it was demonstrated that $\mathrm{Hb}(\beta=0.168, p=0.001)$, BMI $(\beta=0.519, p=0.001)$, and LDL $(\beta=0.331, p=0.000)$ could enter the regression model (adjusted $R^{2}$ for the model $=0.440, p=0.028$ ) (Table 5).

\section{Discussion}

The present study showed a positive association between Hb level and SBP and DBP in males and females with normal glucose tolerance. To our knowledge, this is the first study that investigated the association between $\mathrm{Hb}$ level and blood pressure in a large Chinese 
Xuan et al.: Association of Hemoglobin and Blood Pressure in a Chinese Community-Dwelling Population

Table 5. Correlations of SBP and $\mathrm{DBP}$ with $\mathrm{Hb}$ and heart rate

\begin{tabular}{lcc}
\hline & $\mathrm{Hb}, \mathrm{g} / \mathrm{L}$ & \\
\cline { 2 - 3 }$r$ & $p$ value \\
\hline SBP, mm Hg & 0.075 & $<0.001$ \\
DBP, mm Hg & 0.272 & $<0.001$ \\
Heart rate, bpm & -0.52 & $<0.001$ \\
\hline
\end{tabular}

cohort of healthy individuals. Some earlier cross-sectional studies also focused on the role of iron parameters in blood pressure regulation. Results of these studies are in line with the findings of our study. Göbel et al. [5] found significant correlations between $\mathrm{Hb}$ level and arterial blood pressure in healthy subjects. Some studies have shown that serum ferritin was elevated in males with essential hypertension compared with normotensive males [10]. On a population level, slight fluctuations in blood pressure of only 1-2 $\mathrm{mm} \mathrm{Hg}$ may lead to a substantial difference in cardiovascular mortality and morbidity. Each $20-\mathrm{mm} \mathrm{Hg}$ increase in SBP or $10-\mathrm{mm}$ Hg increase in DBP is associated with a greater than twofold increased risk of cardiovascular death, beginning at $115 \mathrm{~mm} \mathrm{Hg} \mathrm{SBP}$ and $75 \mathrm{~mm} \mathrm{Hg} \mathrm{DBP} \mathrm{[11].}$

More than 290 million Chinese suffer from cardiovascular disease, of whom 270 million suffer from hypertension, and nearly 2 million die prematurely due to high blood pressure each year [12]. Our findings suggest that, in a clinical setting, hypertensive patients may benefit from donating blood by decreasing their $\mathrm{Hb}$ level and, potentially, their blood pressure. The mechanisms that might lead to an elevated blood pressure in individuals with an increased $\mathrm{Hb}$ level are not entirely known. In the past, several biological mechanisms for the association between $\mathrm{Hb}$ and blood pressure have been proposed. First, $\mathrm{Hb}$ is strongly related to arterial stiffness, as measured by pulse wave velocity, which, in turn, increases SBP and DBP [6]. Second, $\mathrm{Hb}$ may be a scavenger of NO produced in the endothelial cells that line the blood vessels, relaxing smooth muscle cells, altering peripheral resistance, and thereby affecting blood pressure. Increased levels of free $\mathrm{Hb}$ may bind to NO, which causes the vessels to constrict and blood pressure to increase [13]. Third, increased Hb levels would be associated with increased blood viscosity; previous observations show that elevated hematocrit and $\mathrm{Hb}$ levels increase blood viscosity and that increased viscosity, partly through an effect on blood pressure, may worsen cardiovascular function. Some studies in hypertensive patients support the role of increased blood viscosity in raising blood pressure [14], but in healthy individuals, the conclusion is not consistent [15]. Increased blood viscosity may cause an increase in NO production and, hence, vasodilatation, through the induction of shear stress, but this is expected to offset increases in blood pressure. Because we did not measure blood viscosity, we do not know if blood viscosity could explain the findings of our study [16]. Fourth, the other plausible mechanisms for the association of $\mathrm{Hb}$ with BPs might be stress [17]. It has been shown that activation of the sympathetic nervous system results in larger increases in hematocrit and $\mathrm{Hb}$, and that the changes in hemoconcentration during mental stress are more highly correlated with changes in cardiovascular measures such as heart rate and blood pressure than physical stressors [17]. Unfortunately, we did not collect data of hematocrit in this research. In this study, we had adjusted heart rate as a confounding factor, and the correlation between HB and BPs persisted. Furthermore, erythropoietin, which was used for the treatment of anemia, has been related to an elevated blood pressure. Proposed underlying mechanisms for vasoconstriction are the presence of erythropoietin receptors on endothelial cells, increased release of serum endothelin 1 from endothelial cells, and expression of serum endothelin 1 mRNA within endothelial cells [7]. Finally, polycythemia is a common blood disease [18]. It is often associated with hypertension, stroke, and even elevated blood pressure as the first symptom $[19,20]$. In addition to blood volume, both $\mathrm{Hb}$ and blood pressure may 
Xuan et al.: Association of Hemoglobin and Blood Pressure in a Chinese

Community-Dwelling Population

be related to the renin-angiotensin-aldosterone system, angiotensin II may play a role in erythropoietin production [21] and may be incited by the sympathetic nervous system, which is known to affect erythropoietin production because subjects with autonomic neuropathy have erythropoietin-responsive anemia [22]. The mechanism of the relationship between $\mathrm{Hb}$ and blood pressure is still unclear, and elucidation requires further research.

The present study has some strengths and limitations. The large data set and the availability of many repeated measurements per person is unique and a strong asset. The extensive amount of repeated data enabled us to estimate very precisely the association between $\mathrm{Hb}$ and SBP and DBP. By using sophisticated statistical methods to analyze repeated measures, we had the opportunity to distinguish the between-person variation from the within-person variation. Furthermore, we were able to adjust for the most important confounding factors such age, sex, and BMI. However, we did not have data on smoking and antihypertensive medication in the present study.

A limitation might be that the present study included only subjects from Shanghai communities, and the results may have been affected by population and regional variables. Further investigations are required in hypertension populations to confirm our hypothesis.

\section{Acknowledgements}

We gratefully acknowledge the invaluable assistance of the physicians of the Department of Geriatrics, Ruijin Hospital, Shanghai, Jiaotong University School of Medicine. The study would not have been possible without their support.

This work was supported by National Nature Science Foundation of China (No. 81500190) and Shanghai Municipal Health Bureau Project (No. 20154y0053) and (201840087).

\section{Statement of Ethics}

The study was approved by Xuhui District community of Shanghai, China. All patients provided written informed consent.

\section{Disclosure Statement}

The authors declare that they have no conflicts of interest to disclose.

\section{References}

1 World Health Organization [Internet]. Cardiovascular diseases (CVDs) factsheet. 2013. Available from http:// www.who.int/news-room/fact-sheets/detail/cardiovascular-diseases-(cvds).

2 Lawes CM, Vander Hoorn S, Rodgers A; International Society of Hypertension. Global burden of bloodpressure-related disease, 2001. Lancet 2008 May;371(9623):1513-1518.

3 Geleijnse JM. Zout, bloeddruk en hart- en vaatziekten. In: Vaartjes I, van Dis I, Visseresn FLJ, Bots, ML. Hart- en vaatziekten in Nederland 2011. Den Haag, Hartsrichting, p 73-90.

4 Staessen JA, Birkenhäger WH. Evidence that new antihypertensives are superior to older drugs. Lancet. 2005 Sep;366(9489):869-71.

5 Göbel BO, Schulte-Göbel A, Weisser B, Glänzer K, Vetter H, Düsing R. Arterial blood pressure. Correlation with erythrocyte count, hematocrit, and hemoglobin concentration. Am J Hypertens. 1991 Jan;4(1 Pt 1):14-9.

6 Kawamoto R, Tabara Y, Kohara K, Miki T, Kusunoki T, Katoh T, et al. A slightly low hemoglobin level is beneficially associated with arterial stiffness in Japanese community-dwelling women. Clin Exp Hypertens. 2012; 34(2):92-8.

7 Kanbay M, Akcay A, Delibasi T, Uz B, Kaya A, Koca C, et al. Comparison of effects of darbepoetin alfa and epoetin alfa on serum endothelin level and blood pressure. Adv Ther. 2007 Mar-Apr;24(2):346-52. 
8 Atsma F, Veldhuizen I, de Kort W, van Kraaij M, Pasker-de Jong P, Deinum J. Hemoglobin level is positively associated with blood pressure in a large cohort of healthy individuals. Hypertension. 2012 0ct;60(4):936-41.

9 Standards of Medical Care in Diabetes-2017: summary of Revisions. Diabetes Care. 2017 Jan;40 Suppl 1:S4-5.

10 Piperno A, Trombini P, Gelosa M, Mauri V, Pecci V, Vergani A, et al. Increased serum ferritin is common in men with essential hypertension. J Hypertens. 2002 Aug;20(8):1513-8.

11 S L. R C, N Q, R P, R C. Age-specific relevance of usual blood pressure to vascular mortality: a meta-analysis of individual data for one million adults in 61 prospective studies. Lancet. 2002;361(9366):1389-90.

12 Yusuf S, Rangarajan S, Teo K, Islam S, Li W, Liu L, et al.; PURE Investigators. Cardiovascular risk and events in 17 low-, middle-, and high-income countries. N Engl J Med. 2014 Aug;371(9):818-27.

13 Cabrales P, Han G, Nacharaju P, Friedman AJ, Friedman JM. Reversal of hemoglobin-induced vasoconstriction with sustained release of nitric oxide. Am J Physiol Heart Circ Physiol. 2011 Jan;300(1):H49-56.

14 Devereux RB, Case DB, Alderman MH, Pickering TG, Chien S, Laragh JH. Possible role of increased blood viscosity in the hemodynamics of systemic hypertension. Am J Cardiol. 2000 May;85(10):1265-8.

15 Vázquez BYS. Blood pressure and blood viscosity are not correlated in normal healthy subjects. Vascular Health Risk Manag. 2012;8:1-6.

16 Lowe GD, Lee AJ, Rumley A, Price JF, Fowkes FG. Blood viscosity and risk of cardiovascular events: the Edinburgh Artery Study. Br J Haematol. 1997 Jan; 96(1):168-73.

17 Allen MT, Patterson SM. Hemoconcentration and stress: a review of physiological mechanisms and relevance for cardiovascular disease risk. Biol Psychol. 1995 Aug;41(1):1-27.

18 Ragione FD, Perrotta S. Polycythemia. Encyclopedia of Cancer. 2016:2394-6.

19 Nand S, Orfei E. Pulmonary hypertension in polycythemia vera. Am J Hematol. 1994 Nov;47(3):242-4.

20 Kang L, Jing C, Chen Y, Zhu Y. Polycythemia vera with hypertension as the first manifestation: clinical analysis of nine cases. Leuk Lymphoma. 2017;26(1):62-4.

21 Freudenthaler SM, Schenck T, Lucht I, Gleiter CH. Fenoterol stimulates human erythropoietin production via activation of the renin angiotensin system. Br J Clin Pharmacol. 1999 Oct;48(4):631-4.

22 Biaggioni I, Robertson D, Krantz S, Jones M, Haile V. The anemia of primary autonomic failure and its reversal with recombinant erythropoietin. Ann Intern Med. 1994 Aug;121(3):181-6. 